\title{
Gram Per Mole of Creatinine
}

National Cancer Institute

\section{Source}

National Cancer Institute. Gram Per Mole of Creatinine. NCI Thesaurus. Code C105486.

A unit of substance concentration defined as the number of grams per mole quantity of creatinine. 